\title{
Angular smoothing and radial regularization of ODF fields: Application on deterministic crossing fiber tractography
}

\author{
K.M. Otto ${ }^{a, *}$, H.-H. Ehricke ${ }^{\text {a }}$, V. Kumar ${ }^{\text {b, } 1}$, U. Klose ${ }^{c}$ \\ a Institute for Applied Computer Science (IACS), University of Applied Sciences, Zur Schwedenschanze 15, \\ 18435 Stralsund, Germany \\ ${ }^{\mathrm{b}}$ Graduate School of Neural \& Behavioural Sciences - International Max Planck Research School, University of Tübingen, \\ Geschwister-Scholl-Platz, 72074 Tübingen, Germany \\ ${ }^{\mathrm{C}}$ MR Research Group, Dptm. of Diagnostic and Interventional Neuroradiology, University Hospital, \\ Hoppe-Seyler-Str. 3, 72076 Tübingen, Germany
}

Received 24 March 2011; received in revised form 31 August 2011; accepted 6 October 2011

Available online 2 November 2011

\author{
KEYWORDS \\ Fiber tracking; \\ HARDI; \\ ODF; \\ Diffusion imaging; \\ Magnetic resonance \\ imaging; \\ MRI; \\ Crossing fibers; \\ Regularization
}

\begin{abstract}
The advent of high angular resolution diffusion imaging (HARDI) has opened up new perspectives for the delineation of crossing and branching fiber pathways. However, image acquisition under clinical conditions with limited measurement time faces the problem of poor spatial and angular resolution and the technique's high susceptibility to noise. In this paper we present a straightforward spatial filter for ODF fields that uses the data-inherent structural information around a voxel as part of a directionally selective method for angular smoothing and radial regularization (ASRR). Especially in regions where fibers cross (multimodal voxels), the method allows us to reduce noise, improve the accuracy of ODF diffusion peaks, and strengthen signals of non-dominant fibers. Moreover, we propose a dynamic scheme in which regularization is applied only to ODFs classified as multimodal. The approach is quantitatively evaluated on synthetic datasets of various configurations. With an in vivo dataset of a human subject, measured under clinical imaging conditions, we demonstrate the method's ability to improve tractography of non-dominant transcallosal fiber pathways and the long fibers of the superior longitudinal fasciculus.
\end{abstract}

(c) 2011 Associazione Italiana di Fisica Medica. Published by Elsevier Ltd. All rights reserved.

\footnotetext{
* Corresponding author. Tel.: +49 3831 456630; fax: +49 3831456687.

E-mail addresses: kay.otto@fh-stralsund.de (K.M. Otto), hans.ehricke@fh-stralsund.de (H.-H. Ehricke), vinod.kumar@med.unituebingen.de (V. Kumar), uwe.klose@med.uni-tuebingen.de (U. Klose).

${ }^{1}$ Current address: University Hospital Aachen, RWTH Aachen, Pauwelsstr. 30, 52074 Aachen, Germany
} 


\section{Introduction}

Diffusion tractography is a technique that aims at the delineation of neural tracts in vivo. Since the microscopic structure of white matter (WM) obstructs diffusion across its local orientation, the detailed characterization of this process with the help of diffusion-weighted magnetic resonance imaging has become a common tool for the reconstruction of WM architecture.

During the last years, numerous methods based upon high angular resolution diffusion imaging (HARDI) [1] have emerged in order to overcome the shortcomings of diffusion tensor imaging (DTI) [2] and to resolve complex white matter architecture such as fiber crossings, branchings and kissings. They can broadly be divided into model-based and model-free approaches. Tuch et al. [1] and Behrens et al. [3] employ a multi-tensor model that is directly applied to the raw diffusion signal, while using different methods of parameterizing and rating the model fit. A more recent tensor decomposition approach, described by Schultz and Seidel [4], is applied to the orientation distribution function (ODF) obtained with q-ball imaging (QBI) [5]. This approach yields good results when applied to the reconstruction of transcallosal fibers with a deterministic tracking algorithm and claims to be able to reliably determine up to three distinct fiber orientations under optimal conditions. The approach of Jansons and Alexander [6] fits a persistent angular structure (PAS) to the diffusion signal, producing a sharp spherical function whose maxima correspond to intravoxel fiber orientations. The framework of spherical harmonics (SH) has inspired various authors to compute a fiber orientation distribution (FOD) as a deconvolution of a monodirectional response function (single-fiber model) from the measured data. The FOD, also being a very sharp spherical function, is a promising method for detecting multiple orientations of intravoxel fiber systems with high angular resolution [7-9]. A linear and model-free reconstruction of the ODF that makes use of the Funk-Radon transform (FRT) of the raw diffusion signal has been proposed by Tuch [5], which could be shown to gain numerical robustness when implemented with the framework of SH $[7,10,11]$. Recently, it has been shown that the original QBI-ODF differs from the true ODF since it uses a linear radial projection rather than the constant solid angle (CSA) during the transform from the 3D probability density function (PDF) to the ODF [12]. The CSA-ODF is properly normalized and much sharper than the QBI-ODF. Though this approach is very promising, it is much more susceptible to noise and may show significant artifacts occurring as strong peaks near the global minimum. Other normalization approaches based upon the marginalization of the radial PDF-integration have also been proposed in order to improve the ODF approximation [13,14].

This paper concentrates on the ODF because of its linearity and model independence. In particular, we evaluate and demonstrate our methods using the original $\mathrm{QBI}$ ODF, which has the advantage of showing a moderate noise sensitivity at acceptable b-values $\leq 2000 \mathrm{~s} / \mathrm{mm}^{2}$ and thus may be still of interest to many researchers. However, its accuracy and ability to resolve small fiber crossing angles is limited, as has been explicitly studied by Zhan and Yang [15]. The situation gets much worse if multiple intravoxel fiber systems cover unequal fractions of the voxel, leading to situations where one fiber system suppresses the occurrence of other fiber systems in the joint ODF. Furthermore, despite its smoothness compared to the normalized versions mentioned above, it is certainly not immune to poor SNR. It is our belief that, especially regarding issues in neurosurgery, techniques with acquisition times of more than 5-10 min make them unsuitable for clinical application and thus multiple acquisitions for SNR optimization are prohibited.

To address the aforementioned problems of the ODF, various post-processing methods have been proposed. For the reduction of noise, a spherical convolution matrix based upon a Gaussian-shaped one-dimensional angular smoothing kernel can be used [5]. Alternatively, limiting the maximum $\mathrm{SH}$-order to a value of 4 largely excludes noise from the approximation. Descoteaux et al. [11] propose incorporating a regularization term (Laplace-Beltrami) into the ODF estimation process in order to reduce the influence of higher-order terms in case they are not needed to approximate the actual diffusion signal. These methods follow an intravoxel (local) processing strategy and usually come at the cost of angular resolution.

Apart from the intravoxel perspective, the presence of neural tracts yields a certain amount of spatial regularity in the shape of neighboring diffusion profiles. While this is the key phenomenon on which tractography relies, postprocessing approaches may use this consistency to arrive at sharper diffusion profiles, preserving or even strengthening peaks that represent non-dominant fibers. Savadjiev et al. $[16,17]$ were among the first to propose a technique that applies to the regularization of HARDI data. The authors introduce the concept of co-helicity, which they use to obtain parameters of curves passing through a given point. Their goal is to improve the directional support within diffusion profiles in terms of local neighborhood. The authors validate their results against ground truth from synthetic data and HARDI data acquired from a biological phantom. Goh et al. [18] extend the estimation of the CSAODF [12] by adding the constraint of nonnegativity to the probability values and further incorporate information from the spatial neighborhood into a convex optimization problem to arrive at spatially regularized ODFs. The very recent approach of Reisert and Kiselev [19] follows a similar strategy based upon constrained spherical deconvolution [8] but does not assume a spherical neighborhood for spatial regularity. Instead, an elongated neighborhood around each gradient direction is used. The geometric approach of Duits and Franken [20] aims at crossingpreserving smoothing of HARDI images using left-invariant diffusion on a 3D Euclidean motion group.

As opposed to the above mentioned approaches that aim at the increase of spatial regularity prior to ODF reconstruction on the basis of CSA-ODFs or FODs, in this paper we describe a method for QBI-ODF regularization as an extension of a local spherical convolution operation into the spatial neighborhood by letting signals from neighboring voxels contribute to the filter process. We follow a threefold strategy: (1) smoothing in order to suppress noise, (2) regularization in order to improve accuracy of maxima detection and (3) sharpening in order to strengthen signals of non-dominant fiber systems. In particular, the last two 
goals apply to multimodal voxels containing fiber crossings. Apart from the common usage of the term "regularization" in mathematics, we use it to denote the increase of spatial regularity (compare to Savadjiev et al. [16] and Goh et al. [18]), between neighboring ODFs. The method presented here is also a generalization and extension of an ad-hoc approach we described in an earlier conference publication [21,22], which lacked a quantitative analysis. We evaluate our method quantitatively using synthetic data and show its potential to improve deterministic tractography of the corpus callosum and the superior longitudinal fasciculus with the example of in vivo data from a human brain.

\section{Material and methods}

\section{ODF reconstruction}

For the reconstruction of ODFs from the raw diffusion data, we use the spherical harmonics approach described in [11]. In order to be able to delineate fiber crossings even at low angles and avoid unnecessary loss of angular resolution, we choose a high SH order of 8 for all ODF reconstructions. Depending on the experiment, we optionally enable Laplace-Beltrami regularization with $\lambda=0.006$, which, as the authors point out, is a suitable value over a large range of SNR and b-values. Generally, we use a set of 606 ODF reconstruction directions evenly distributed on a hemisphere that we obtained from an electrostatic repulsion simulation.

\section{Synthetic data - crossing fibers (CF)}

Synthetic diffusion datasets $(17 \times 17 \times 6$ voxels, $2 \mathrm{~mm}$ isotropic voxel size) containing two straight fibers (each $5 \mathrm{~mm}$ in diameter) crossing each other at various angles $\alpha_{c}$ were generated using a partial volume model similar to the one described in [23]. The synthetic diffusion signal for a particular diffusion gradient was computed as a mixture of fully anisotropic components representing fibers, modeled by diffusion tensors with two eigenvalues being zero, and a single isotropic component (fully isotropic tensor) with a volume fraction $\omega_{\text {iso }}$ representing free water. Noise was modeled as complex Gaussian noise with a standard deviation of $\sigma$ with respect to the b0-signal $(\mathrm{SNR}=1 / \sigma)$. In voxels containing fractions of both fibers, it was possible to modulate the distribution of corresponding volume fractions by a parameter $d_{f}=$ dominance of fiber $1 /$ dominance of fiber2, allowing the simulation of one fiber dominating the other in terms of signal contribution. Further parameters we chose were diffusivity $d=0.0015 \mathrm{~mm}^{2} / \mathrm{s}$ and the baseline signal $S_{0}=100$. In voxels completely occupied by fiber segments, we ensured a minimum of $\omega_{\text {iso }}=0.35$. The aforementioned parameters were chosen such that the signals obtained from monomodal voxels in the corpus callosum of the human subject dataset could be closely reproduced in monomodal voxels of the synthetic dataset. Consequently, we used the same 64 diffusion gradients and $b=2000 \mathrm{~s} / \mathrm{mm}^{2}$ as in our human subject study for the generation of synthetic diffusion data.
We generated a total of $112 \times 100$ synthetic datasets for various crossing angles $\alpha_{c}(25,30, . .90)^{\circ}$, SNR $(10,20,35$, $1000)$, and $d_{f}(0.5,1)$. For every of the 112 combinations of $\alpha_{c}$, SNR, and $d_{f}$ (dataset configuration), we computed 100 ODF datasets in order to quantify the noise-related spreading (mean, standard deviation) of metrics we used to evaluate our approach.

\section{Synthetic data - crossing fibers with curvature (CFC)}

Using the same method as described above, a total of 24 synthetic ODF datasets $(55 \times 54 \times 13$ voxels, $2 \mathrm{~mm}$ isotropic voxel size) containing two straight fibers (each $5 \mathrm{~mm}$ in diameter) crossing at three different angles $\alpha_{c}\left(45^{\circ}, 55^{\circ}\right.$, $\left.75^{\circ}\right)$ were generated for various SNR $(10,20,35,1000)$, and $d_{f}(0.5,1)$. For the first fiber an additional spiral part was modeled that started after crossing the second fiber and decreased linearly in diameter from $32 \mathrm{~mm}$ down to $10 \mathrm{~mm}$.

\section{In vivo data - human subject}

Diffusion data (56 slices, image matrix $108 \times 108$ ) from the brain of a healthy 29-year-old human female was acquired on a 3-T Trio MR scanner (Siemens Medical Systems, Erlangen, Germany) using a spin-echo echo-planar diffusion-weighted sequence $(T R=8000 \mathrm{~ms}, T E=105 \mathrm{~ms})$ with 64 diffusionencoding gradients, a b-value of $2000 \mathrm{~s} / \mathrm{mm}^{2}$, and an isotropic voxel size of $2 \mathrm{~mm}$. Measurement time was approximately $8.7 \mathrm{~min}$. SNR was measured with the standard two-ROI (region of interest) approach, resulting in an average value of 50 (b0-images) resp. 13 (gradient images). Note that these values can give only an approximation and may be inaccurate because there is a dependency on the ROls chosen.

\section{Multi-directional streamline tracking}

In order to perform experiments of tract reconstruction, we employed a multi-directional deterministic streamline tracking approach applied to the dataset of ODFs. The algorithm is similar to the one described by Wedeen et al. [24] and may be outlined here in short: During tract propagation, the ODF is obtained at each step from tri-linear interpolation. Then, the direction of the diffusion peak that best fits the previous propagation direction is selected. If this direction does not exceed a maximum deviation angle $\alpha_{\max }$ from the previous step, it is used for the next propagation step. Otherwise, or if the FA value falls below a minimum of $f a_{\text {min }}$, tracking stops. In our experiments, tract propagation was performed with $\alpha_{\max }=40^{\circ}, f a_{\min }=0.2$, a step size of $s=1 \mathrm{~mm}$ and 4-th order Runge-Kutta integration.

We decided to evaluate our results using a straightforward, deterministic tracking technique because of its practicability in terms of computational effort and interpretation of tracking results. The low detection rate and accuracy of local maxima at critical crossing angles and unequal fiber volume fractions are well-known problems of the diffusion ODF that show up clearly as tract truncations and deviations inside crossing regions. Thus, since our method is focused on the sharpening of ODFs in such areas in order to improve the detection of local maxima, its 
impact can easily be demonstrated and understood with deterministic tracking.

\section{Angular smoothing and radial regularization (ASRR)}

Looking at an ODF as a spherical function, we introduce an angular dimension in terms of deviation between two reconstruction directions. In order to consider the spatial neighborhood around an ODF, we further add a radial dimension in terms of distance from the voxel center. While intravoxel noise suppression as described by Tuch [5] can be seen as a convolution of the ODF with a filter kernel living on the surface of the ODF, we enhance the space of contributing values from neighboring ODFs with the radial dimension. This leads us to a spatial filter kernel whose shape determines the topology of samples drawn to compute the filter result. At least it lets us control the magnitude of both angular smoothing and radial regularization.

\section{Diffusion profile}

Let $\Psi(\vec{p}, \vec{d})$ be an ODF at the location $\vec{p}$ within the data volume and $\vec{d} \in R ;|\vec{d}|=1$ a vector from the set $R$ of reconstruction directions.

\section{Symmetry}

Since we cannot measure the polarity of diffusion in a particular direction, an ODF is assumed to be a pointsymmetric function:

$\Psi(\vec{p}, \vec{d})=\Psi(\vec{p},-\vec{d})$

This makes the existence of both a direction $\vec{d}$ and its opposite $-d$ within the set $R$ redundant. Thus, $R$ is assumed to contain directions distributed on a hemisphere.

\section{Angular filter kernel}

Let $\vec{d}_{f} \in R$ be the direction whose value $\Psi\left(\vec{p}, \vec{d}_{f}\right)$ is to be filtered, $\quad \vec{d}_{j} \in R$ any direction from the set $R$, and $\alpha_{f j}=\operatorname{acos}\left(\left|\vec{d}_{f} \cdot \vec{d}_{j}\right|\right)$ the angle $\in\left[0, \frac{\Pi}{2}\right]$ between both directions. An angular filter kernel can then be expressed as a one-dimensional function $\Omega\left(\alpha_{f j}\right)$ that decays with increasing angle $\alpha_{f j}$. Following the proposal of an intravoxel smoothing filter [5] and in accordance with commonly used filter kernels in image processing, we choose $\Omega$ to be a Gaussian-shaped function:

$\Omega\left(\alpha_{f j}\right)=\exp \left(-\frac{\alpha_{f j}^{2}}{\sigma_{a}^{2}}\right)$

The parameter $\sigma_{a}$ controls the width of the kernel and thus the amount of angular smoothing.

\section{Radial filter kernel}

Let $\rho$ be the Euclidean distance to the position $\vec{p}$ of the diffusion profile. A radial smoothing kernel can then be expressed as a one-dimensional function $\Xi(\rho)$ that decays with increasing distance $\rho$. Here, we also choose $\Xi$ to be a Gaussian-shaped function:
$\Xi(\rho)=\exp \left(-\frac{\rho^{2}}{\sigma_{r}^{2}}\right)$

The parameter $\sigma_{r}$ controls the width of the kernel and thus the influence of neighboring diffusion profiles.

\section{Spatial filter kernel}

A spatial filter kernel can be expressed as a product of the angular and the radial filter kernel:

$\Upsilon\left(\alpha_{f j}, \rho\right)=\Omega\left(\alpha_{f j}\right) \Xi(\rho)=\exp \left(-\frac{\alpha_{f j}^{2}}{\sigma_{a}^{2}}-\frac{\rho^{2}}{\sigma_{r}^{2}}\right)$

Fig. 1 illustrates the shape of such a kernel as a twodimensional contour plot. The combination of angular and radial filter kernels yields a spatial function with a Gaussian decay along the deviation $\alpha_{f j}$ from the direction to be filtered, $\vec{d}_{f}$, and the distance $\rho$ from the voxel center located at the center of the plot. Note that the filter is directionally selective, i.e., only values of directions from neighboring ODFs that coincide with the corresponding radial (reconstruction direction) $\vec{d}_{j}$ contribute to a filtered ODF value.

\section{Filtering}

For every direction $\vec{d}_{i}$ from the set $R$, the filtered value $\tilde{\Psi}\left(\vec{p}, \vec{d}_{i}\right)$ at location $\vec{p}$ can be computed in the following manner:

$\tilde{\Psi}\left(\vec{p}, \vec{d}_{i}\right)=\frac{1}{Z_{i}} \sum_{j} \sum_{\rho=-\rho_{\max }}^{+\rho_{\max }} \Upsilon\left(\alpha_{i j}, \rho\right) \Psi\left(\vec{p}+\rho \overrightarrow{\boldsymbol{d}}_{j}, \overrightarrow{\boldsymbol{d}}_{j}\right) ; \overrightarrow{\boldsymbol{d}}_{i}, \overrightarrow{\boldsymbol{d}}_{j} \in R$

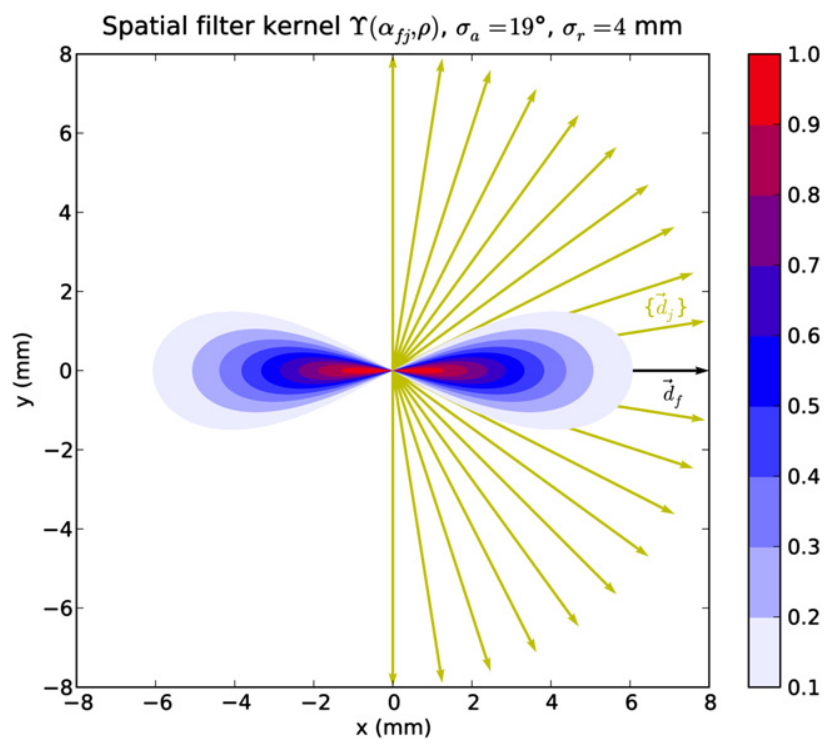

Figure 1 Two-dimensional contour plot of the spatial filter kernel $\Upsilon\left(\alpha_{f j}, \rho\right)$. With increasing angle $\alpha_{f j}$ and distance $\rho$ from the voxel center at $(x=0 / y=0)$, the resulting value decays. Note that the actual shape of $Y$ is three-dimensional. The yellow arrows illustrate the reconstruction directions $\vec{d}_{j}$, which usually are distributed on a hemisphere. The black arrow corresponds to the direction $\vec{d}_{f}$ to be filtered. 
with a normalization constant $Z_{i}$ :

$Z_{i}=\sum_{j} \sum_{\rho=-\rho_{\max }}^{+\rho_{\max }} \Upsilon\left(\alpha_{i j}, \rho\right)$

The limit $\rho_{\max }$ defines the extent of the voxel's neighborhood considered for filtering. It can be derived from $\Xi$ as parameter where the sample's contribution falls below a certain threshold, or it may be directly parameterized by the user in order to gain full control over radial weighting.

If $\sigma_{r}$ is chosen to be close to zero $\left(\sigma_{r} \rightarrow 0 \mathrm{~mm}\right)$, ASRR behaves like Tuch's intravoxel smoothing. If we increase the width of the radial smoothing kernel, neighboring diffusion profiles contribute more and more to the overall filtering process and the regularization effect grows. To preserve point symmetry of $\tilde{\Psi}$, we have to let $\rho$ run between $-\rho_{\max }$ and $+\rho_{\max }$. Thus, we always look forward and backward when we integrate neighboring profiles into a filtered value for a particular direction. We use a step size of $s_{\rho}=1 \mathrm{~mm}$ in order to iterate along each radial.

All experiments in this work where regularization was enabled have been performed with a value of $\sigma_{r}=4 \mathrm{~mm}$. With such a parameterization, the maximal distance at which the weight of neighboring samples falls below a threshold of 0.1 is about $6 \mathrm{~mm}$. Because the contribution of samples being more distant is rather low and computation time increases with $\rho_{\max }$, we chose $\rho_{\max }=6 \mathrm{~mm}$.

Fig. 2 shows the influence of the smoothing $\left(\sigma_{a}\right)$ and regularization $\left(\sigma_{r}\right)$ parameters on the shape of filtered ODFs from nine voxels in the crossing region of a synthetic CF dataset with $\mathrm{SNR}=10, \alpha_{c}=90^{\circ}$ overlaid onto an FA slice. The volume fraction of the horizontal fiber system is only half of the vertical fibers $\left(d_{f}=0.5\right)$. The yellow lines indicate detected ODF maxima. The symbols $\oplus$ indicate additional maxima found in ODFs ASRR was applied to, whereas maxima suppressed by too strong smoothing are indicated by the symbols $\Theta$. Image (a) shows the strong influence of noise on the unfiltered ODFs and detected maxima. Noise reduction may be performed solely by angular smoothing with increasing $\sigma_{a}$ (images $\mathrm{a}-\mathrm{c}$ ). The drawback of this approach is twofold: (1) Diffusion peaks of the nondominant horizontal fiber system may easily be smoothed out (image c). (2) The orientations of remaining maxima are not well aligned with the horizontal direction, which is still a consequence of noise. Unfortunately, lower-frequency portions of noise that cannot be sufficiently suppressed by intravoxel smoothing distort the shape of ODFs.

When we perform radial regularization only $\left(\sigma_{a} \rightarrow 0^{\circ}\right.$, $\sigma_{r}>0$ ), the shape of the ODFs can be significantly sharpened and the orientations of detected ODF maxima are more aligned with the underlying fiber architecture (images a, d, g). Nevertheless, noise suppression may not be enough. Furthermore, since ASRR is designed to improve tractography through crossing regions and the neighborhood is sampled along straight radials, regularization may easily be overdone in the presence of strongly bending fiber systems, inducing a linearizing effect on reconstructed fiber trajectories. Choosing $\sigma_{a}$ to be too small and/or $\sigma_{r}$ too large may enhance this effect. In practice, a good tradeoff between angular smoothing and radial regularization has to be found (images $e, h$ ) that depends not only on SNR but also on the fiber architecture to be delineated.

\section{FA threshold}

When gathering samples from the neighborhood, we have to avoid the incorporation of voxels that may not represent white matter. A commonly used method to mask out such voxels is to apply a threshold to the FA values. For ASRR, we used the same threshold $f a_{\min }=0.2$ as in our tractography experiments.

\section{Dynamic ASRR}

Unlike smoothing, the value of regularization as done by ASRR applies mostly to regions of multimodal voxels that represent fiber crossings. As addressed in the previous section, too strong regularization may have a linearizing effect on bending fiber pathways to be reconstructed with the streamline approach. In order to reduce this effect, regularization can simply be discarded $\left(\sigma_{r} \rightarrow 0 \mathrm{~mm}\right)$ for monomodal ODFs. As opposed to using ANOVA [25] or a Bayesian Framework [23] for the distinction between monomodal and multimodal diffusion, we choose a simpler approach with the advantage of directly working on the reconstructed ODFs used for tracking. Once for a dataset, we first search for the voxel with the highest FA value $f a_{\max }$. We take the ODF $\Psi_{m}$ in this voxel to be the single fiber model ODF and assume all voxels with an FA value of at least $0.95 * f a_{\max }$ to be monomodal. Voxels with a lower FA value are assumed to be monomodal if a measure of similarity with the single fiber model ODF exceeds a certain threshold.

In order to obtain the threshold, we determine all $N$ ODFs $\Psi_{i}$ with an FA value $\geq 0.95 * f a_{\text {max }}$ and compute their similarities $S\left(\Psi_{m}, \Psi_{i}\right) ; i=1 . . N$ with the single fiber model $\Psi_{m}$. The threshold $S_{t}$ above which a voxel is classified as monomodal is calculated as the mean of the similarities $S\left(\Psi_{m}, \Psi_{i}\right)$ subtracted by the standard deviation:

$S_{t}=T *\left(\operatorname{mean}\left(S\left(\Psi_{m}, \Psi_{i}\right)\right)-\operatorname{stddev}\left(S\left(\Psi_{m}, \Psi_{i}\right)\right)\right)$

The parameter $T$ can be specified by the user to tweak the classification threshold. Especially in the presence of noise, it may be appropriate to lower $T$ since monomodal ODFs could erroneously be classified as multimodal. In our experiments with synthetic datasets at various noise levels and fiber-crossing angles, we found $T=0.98$ to be a good choice for the whole range of configurations.

Since $\Psi_{m}$ and $\Psi_{i}$ can be oriented differently, $S\left(\Psi_{m}, \Psi_{i}\right)$ needs to be rotational invariant. Furthermore, the similarity metric should be sensitive to differences in the ODF shapes. In order to achieve this, we first scale each ODF value $\Psi(\vec{d}) ; \vec{d} \in R$ by the minimal angular deviation of its corresponding reconstruction direction $\vec{d}$ from the direction $\vec{d}_{\max }$ of the global ODF maximum: $\Psi^{s}(\vec{d})=\left|\vec{d} \cdot \vec{d}_{\max }\right| * \Psi(\vec{d})$. Next, we compute the Pearson correlation between $\Psi_{m}^{s}$ and $\Psi_{i}^{s}$, but based upon the sorted values of each function. To understand why both scaling and sorting are needed to meet the requirements of the similarity metric, one can start with two identical ODFs whose orientations differ by an arbitrary angle. Since the set of values is equivalent in both ODFs, the orientation information is encoded solely in the order of 


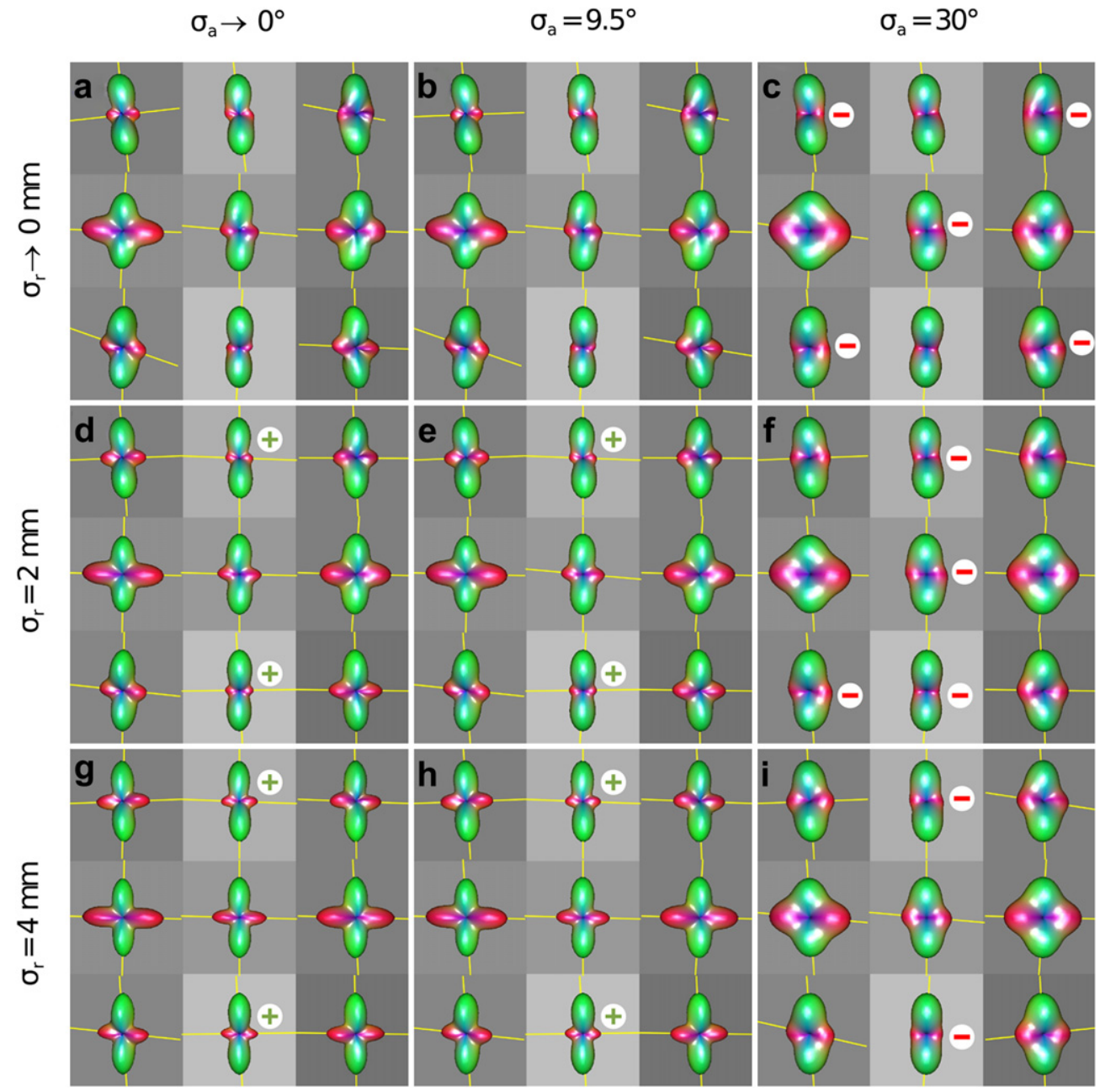

Figure 2 Nine voxels from a $90^{\circ}$-crossing region of a synthetic dataset at SNR $=10$. Volume fraction of the horizontal fiber system: 0.5. Yellow lines indicate detected maxima. The symbols $\oplus$ indicate additional maxima found in ODFs ASRR was applied to. The symbols $\odot$ indicate maxima suppressed by too strong smoothing. (For interpretation of the references to color in this figure legend, the reader is referred to the web version of this article.)

the values (the mapping of values to the fixed set of reconstruction directions). Sorting the values of both ODFs will result in two identical lists. Hence, the correlation of these two lists will be 1 , while the correlation of the original values will not. Now, if we assume one monomodal and one multimodal ODF with the latter representing a $90^{\circ}$-crossing of fibers, it becomes obvious that the preliminary scaling of ODF values with respect to the direction of the global maximum further decreases the similarity between the sorted value lists. This is due to the fact that the scaling imprints additional information - the angular distribution of ODF values around $\vec{d}_{\max }-$ to the sorted values.

We would like to point out that it is beyond the scope of this paper to suggest an improved methodology to discriminate between mono- and multimodal diffusion and to investigate its behavior further. The approach described here was chosen because of its computational effectiveness since it works on exactly the same data used for tracking and does not require the calculation of multiple ADC profile approximations or Markov Chain MonteCarlo (MCMC) sampling required for Bayesian estimation. Thus, it can also easily be applied to interpolated ODFs during streamline tracking. Nevertheless, the classification performance of all methods suffers from poor SNR, which may limit the effectiveness of dynamic ASRR.

\section{Implementation details}

For the interpolation of an ODF at non-discrete locations, we use tri-linear interpolation of each separate ODF value.

Filtering a single ODF with ASRR $\left(\rho_{\max }=6 \mathrm{~mm}\right.$, radial sampling stepwidth $=1 \mathrm{~mm}$ ) takes about $200 \mathrm{~ms}$ (Intel ${ }^{\circledR}$ Core 2 Duo dual-core processor with $2.4 \mathrm{GHz}$ per core, $J_{a v a}{ }^{\mathrm{TM}} 1.6$ Runtime Environment, 606 reconstruction directions). By using a threshold $b 0_{\min }=20$ for the $b 0$-value, the whole human subject dataset can be filtered in approximately $9 \mathrm{~h}$. A lower number of ODF reconstruction directions, for example 406 or 246, decreases computation time to approximately 4 or $1.5 \mathrm{~h}$, respectively. A higher 
threshold, radial stepwidth or a more optimized implementation may further reduce time consumption.

\section{Detection of diffusion peaks}

To detect diffusion peaks within an ODF, we search for local maxima. An ODF value is identified as local maximum if (1) it reaches at least a certain fraction $t_{p}$ of the minimum-tomaximum interval of the ODF, (2) all neighboring values on the tessellated ODF surface have a smaller value and (3) its direction does not deviate less than an angle of $\alpha_{\min }$ from all previously found peaks.

By setting $\alpha_{\min }$ to an appropriate value, additional peaks that may be related to noise can be excluded from the environment of previously found peaks. Since it is known that with a b-value of $2000 \mathrm{~s} / \mathrm{mm}^{2}$, it is impossible to resolve crossings below an angle of approx. $50^{\circ}$ from an ODF obtained with QBI, we chose a value of $30^{\circ}$ for the tractography experiments, which is still far below the critical angle. Moreover, we set $t_{p}=0.15$ in order to exclude peaks that we assume are most likely related to noise. Except for the minimum angle constraint, this approach is similar to the ones described by Wedeen et al. [24] and Descoteaux et al. [9].

\section{Results}

\section{Quantitative evaluation of ASRR on synthetic data}

\section{Parameter estimation}

The parameterization of ASRR has a strong impact on the shape of a filtered ODF and hence on the success and accuracy of maxima detection. The amount of regularization is controlled mainly by $\sigma_{r}$ and should be adapted to the expected architecture of the fiber system to be reconstructed. This applies especially to its shape (curvature) and its relations to other fiber systems (size of possible crossing regions). As mentioned earlier, $\sigma_{r}$ should be chosen carefully since the delineation of strongly bending fibers may deteriorate with high values. Thus, we generally fixed $\sigma_{r}$ to a value of $4 \mathrm{~mm}$ when regularization was enabled. We chose this value to be slightly smaller than the size of the crossing region $(5 \mathrm{~mm}$ in diameter) in the synthetic datasets at $\alpha_{c}=90^{\circ}$.

The choice of $\sigma_{a}$ influences noise suppression most. With regard to noise suppression, choosing the appropriate value for $\sigma_{a}$ should depend on SNR. In his original paper, Tuch [5] suggests the parameterization of angular smoothing based upon visual inspection of the ODFs as an ad-hoc procedure, which is a common strategy in image processing. However, for a quantitative evaluation of ASRR we need to specify $\sigma_{a}$ on the basis of a reproducible criterion that can be used in all experiments with synthetic data at specific SNR levels.

For the determination of the best value $\tilde{\sigma}_{a}$, we computed the Pearson correlation $C$ between the noise-free ODF $\Psi$ and the noisy ODF $\operatorname{ASRR}\left(\Psi_{\text {noise }}\right)$ filtered with ASRR with $\sigma_{a}(0$, $1, . .45)^{\circ}$ without regularization $\left(\sigma_{r} \rightarrow 0 \mathrm{~mm}\right)$ for $K=10$ different two-fiber-crossing angles $\beta_{c}(0,10, . .0 .90)^{\circ}$ under various SNR $(10,20,35,1000)$ and fiber dominance ratio $d_{f}$ $(0.5,1)$. As a result, for each combination of SNR and $d_{f}$ we obtained 10 different functions $C_{k}\left(\sigma_{a}\right) ; k=1$..K, one for each crossing angle $\beta_{c}$. We then computed the mean $\tilde{C}\left(\sigma_{a}\right)$ of the 10 functions and determined its argument $\tilde{\sigma}_{a}$ where the function reached its maximum. The results are shown in Table 1. As expected, the best value for $\sigma_{a}$ is strongly correlated with SNR and a low SNR forces us to use much stronger angular smoothing than does a high SNR. Obviously, the influence of $d_{f}$ is rather low. Only for $S N R=20$, there is a slight difference of $1^{\circ}$ in the determined values for $\tilde{\sigma}_{a}$. Thus, we took a value of $\tilde{\sigma}_{a}=13^{\circ}$ for both $d_{f}$ in all further experiments with $\mathrm{SNR}=20$.

Since the smoothing effect of ASRR is mainly, but of course not solely, controlled by $\sigma_{a}$, the question of how far we can assign the results of this intravoxel smoothing experiment to the parameterization of $\sigma_{a}$ when performing full ASRR $\left(\sigma_{r}=4 \mathrm{~mm}\right)$ is difficult to answer. Here, many more samples contribute to a filtered value than in the case of intravoxel smoothing. A clue might be given by the total sum of weights assigned to samples contributing to a single ODF value during the filtering process: In case of $\sigma_{r}=4 \mathrm{~mm}$, the sum of weights is approximately 4 times larger than in the case of $\sigma_{r} \rightarrow 0 \mathrm{~mm}$. Hence, an ad-hoc solution might be to use only a half or a quarter of $\tilde{\sigma}_{a}$ for full ASRR in order to not overdo smoothing.

\section{Evaluation of the success rate and accuracy of ODF maxima detection}

With the help of the synthetic CF datasets, we determined the accuracy of ODF maxima and the rate at which exactly two maxima could be detected (success rate). Therefore, we defined a spherical region of $4 \mathrm{~mm}$ in diameter which we positioned exactly in the center of the crossing region of each CF dataset. With an isotropic step size of $0.5 \mathrm{~mm}$, the crossing region was filled with sampling points, leading to a total of 251 points. For every combination of $\alpha_{c}$, SNR, and $d_{f}$ (dataset configuration), the ODF for each sampling point was interpolated in each of the 100 ODF datasets and its maxima were detected. Thus, we computed $251 \times 100$ sets of ODF maxima for each dataset configuration. From each of these sets, an accuracy metric for the detected maxima $\vec{d}_{p 1}$ and $\vec{d}_{p 2}$ was calculated if exactly two maxima could be found. In order to quantify accuracy, the angular deviation (angular error $E$ ) between the two real fiber orientations $\vec{r}_{1}$ and $\vec{r}_{2}$ and the corresponding maxima $\vec{d}_{p 1}$ and $\vec{d}_{p 2}$ was computed as the mean of both deviations: $E=\frac{1}{2}\left(\operatorname{acos}\left(\left|\vec{r}_{1} \cdot \vec{d}_{p 1}\right|\right)+\operatorname{acos}\left(\left|\vec{r}_{2} \cdot \vec{d}_{p 2}\right|\right)\right)$. Finally, the mean of all $251 \times 100$ angular errors was taken as overall angular error $\tilde{E}$. Additionally, the standard deviation was computed. The experiment was repeated under different conditions (ODF filters/ODF reconstructions):

1. Unfiltered $(\lambda=0)$

2. Local (intravoxel) smoothing $\left(\sigma_{r} \rightarrow 0 \mathrm{~mm}, \sigma_{a}=\widetilde{\sigma}_{a}, \lambda=0\right)$

3. Laplace-Beltrami regularization $(\lambda=0.006)$

Table 1 Best parameter $\tilde{\sigma}_{a}$ for different SNR and fiber dominance ratio $d_{f}$.

\begin{tabular}{lllllllll}
\hline SNR & 10 & & 20 & & 35 & \multicolumn{2}{c}{1000} \\
\hline$d_{f}$ & 0.5 & 1.0 & 0.5 & 1.0 & 0.5 & 1.0 & 0.5 & 1.0 \\
$\tilde{\sigma}_{a}$ & $19^{\circ}$ & $19^{\circ}$ & $13^{\circ}$ & $14^{\circ}$ & $9^{\circ}$ & $9^{\circ}$ & $2^{\circ}$ & $2^{\circ}$ \\
\hline
\end{tabular}



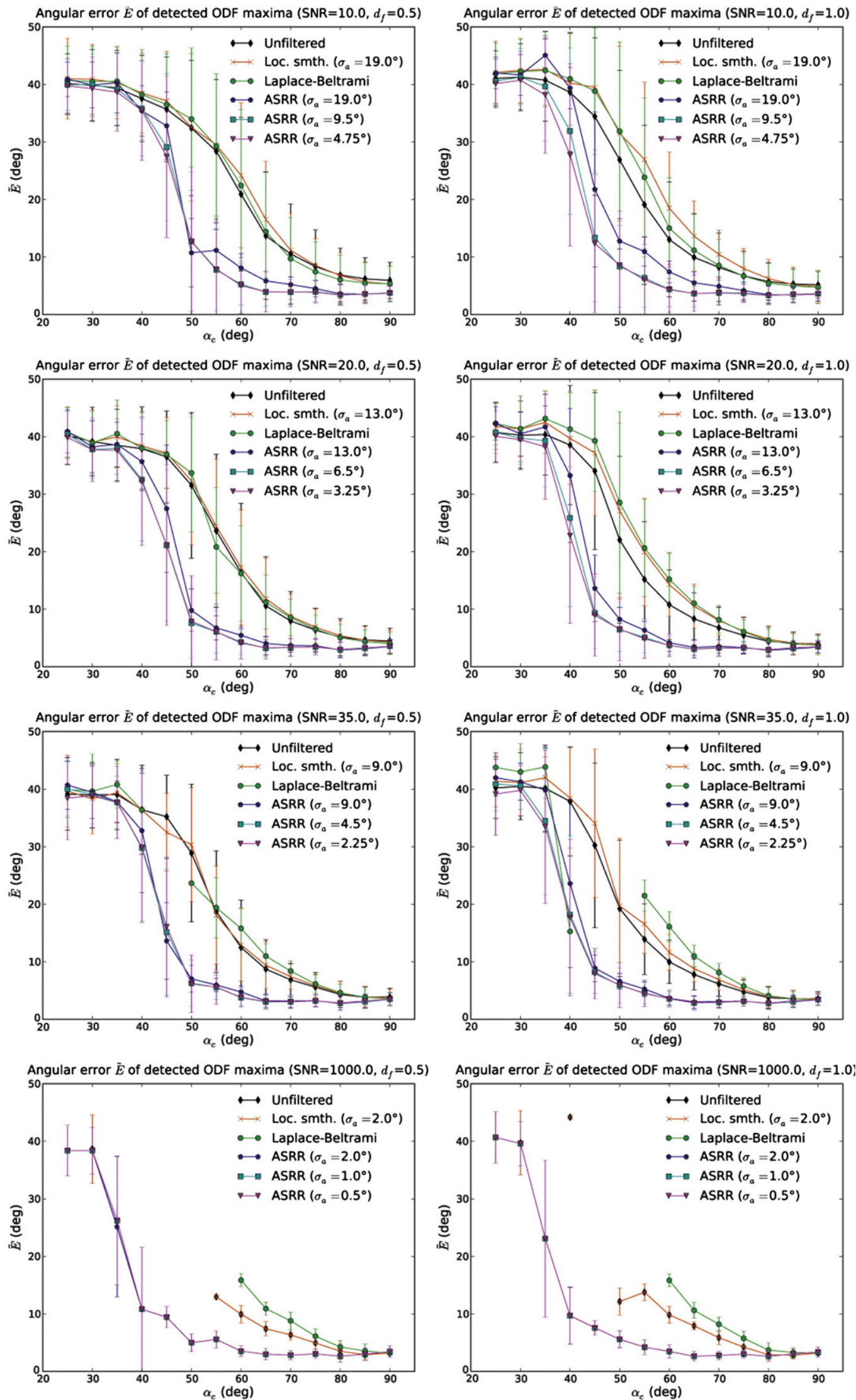

Figure 3 Angular error $\tilde{E}$ between real fiber orientations and detected ODF maxima for different dataset configurations (SNR, fiber dominance ratio $d_{f}$, fiber-crossing angle $\alpha_{c}$ ). For each configuration, the angular error has been computed for different ODF filters/ODF reconstructions. 

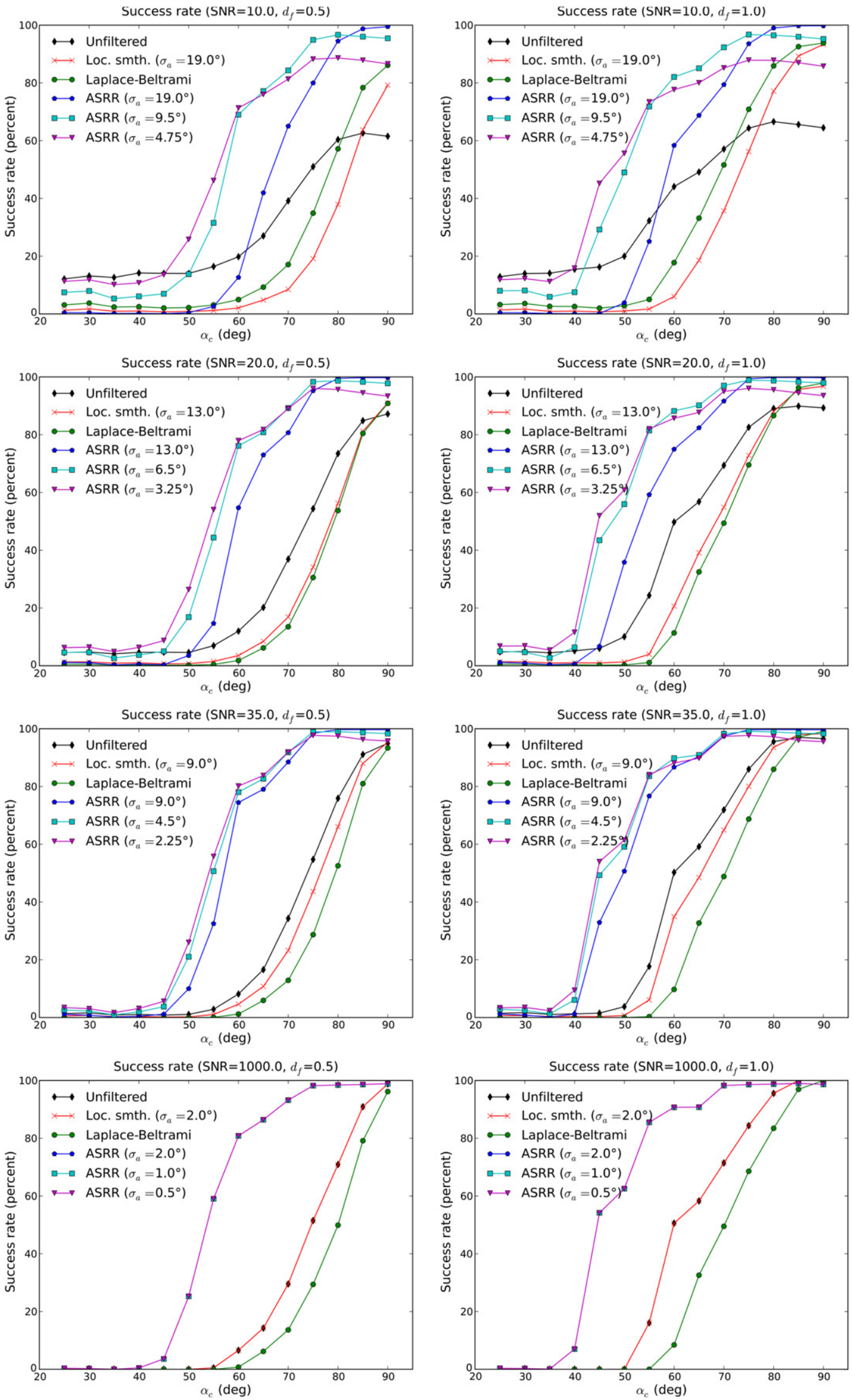

Figure 4 Success rate of finding exactly two ODF maxima for different dataset configurations (SNR, fiber dominance ratio $d_{f}$, fiber-crossing angle $\alpha_{c}$ ). For each configuration, the success rate has been computed for different ODF filters/ODF reconstructions. 
4. $\operatorname{ASRR}\left(\sigma_{r}=4 \mathrm{~mm}, \sigma_{a}=\widetilde{\sigma}_{a}, \lambda=0\right)$

5. ASRR $\left(\sigma_{r}=4 \mathrm{~mm}, \sigma_{a}=\tilde{\sigma}_{a} / 2, \lambda=0\right)$

6. ASRR $\left(\sigma_{r}=4 \mathrm{~mm}, \sigma_{a}=\widetilde{\sigma}_{a} / 4, \lambda=0\right)$

Figs. 3 and 4 show the results of the experiments. It can be seen that the angular error $\tilde{E}$ decreases with increasing fiber-crossing angle $\alpha_{c}$, reaching a minimum value in the range of $4-7^{\circ}$ at $\alpha_{c}=90^{\circ}$. As expected, the success rate grows with increasing $\alpha_{c}$. When looking at the almost noise-free datasets $(S N R=1000)$, it becomes obvious that the critical fiber-crossing angle that allows two distinct ODF maxima to be detected lies in a range of $55-60^{\circ}$ when ASRR is not applied. If one fiber dominates the other $\left(d_{f}=0.5\right)$, it is generally more difficult to detect the non-dominant one, which is clearly reflected in the success rate. If SNR gets worse, small perturbations on the surface of the ODFs seem to ease the satisfaction of the local maximum constraint and thus to find additional diffusion peaks, especially at low fiber-crossing angles. It should not be concluded that low SNR improves the situation. Instead, the susceptibility of ODF maxima detection to noise induces a tendency to produce wrong maxima with a large angular error. This is clearly shown in the plots: If the fiber-crossing angle ranges between $25^{\circ}$ and $55^{\circ}$, the angular error of the ODFs not filtered with ASRR is rather high $\left(25-55^{\circ}\right.$ at $\left.S N R=10\right)$ and the standard deviation is large. Obviously, filtering the ODFs with ASRR causes the angular error to drop significantly. While in a range of $50-60^{\circ}$, the advance of ASRR is greatest (approximately $10-20^{\circ}$ ), the accuracy of all methods converges at $\alpha_{c}=90^{\circ}$. However, unlike the other methods, ASRR seems to produce more stable results in the range of $\alpha_{c}=50-90^{\circ}$, which can be seen in the standard deviation and the much lower decay of the angular error toward $\alpha_{c}=90^{\circ}$. Depending on SNR, ASRR shows no improvements below a fiber-crossing angle of $40-50^{\circ}$.

With regard to the best angular smoothing value for ASRR, it can be concluded that a value of $\sigma_{a}=\widetilde{\sigma}_{a} / 2$ or $\sigma_{a}=\widetilde{\sigma}_{a} / 4$ results in a lower angular error and a higher success rate than $\sigma_{a}=\widetilde{\sigma}_{a}$. This is an expected outcome since $\tilde{\sigma}_{a}$ has been determined for local smoothing (see previous section), and the smoothing effect of full ASRR is stronger than local smoothing. Generally, the chance to overdo smoothing and to deteriorate success rate and angular error seems to be high in the range of fiber-crossing angles where the detection of both fiber orientations is indeed possible. Only in the proximity of $90^{\circ}$, as can be clearly observed at low SNR, does smoothing improve the situation.

To conclude the results of this experiment, it can be said that (1) ASRR has no advancing effect at fibercrossing angles below $40-50^{\circ}$, depending on SNR. (2) The accuracy and success rate of ODF maxima detection is significantly improved by ASRR in areas where fibers cross
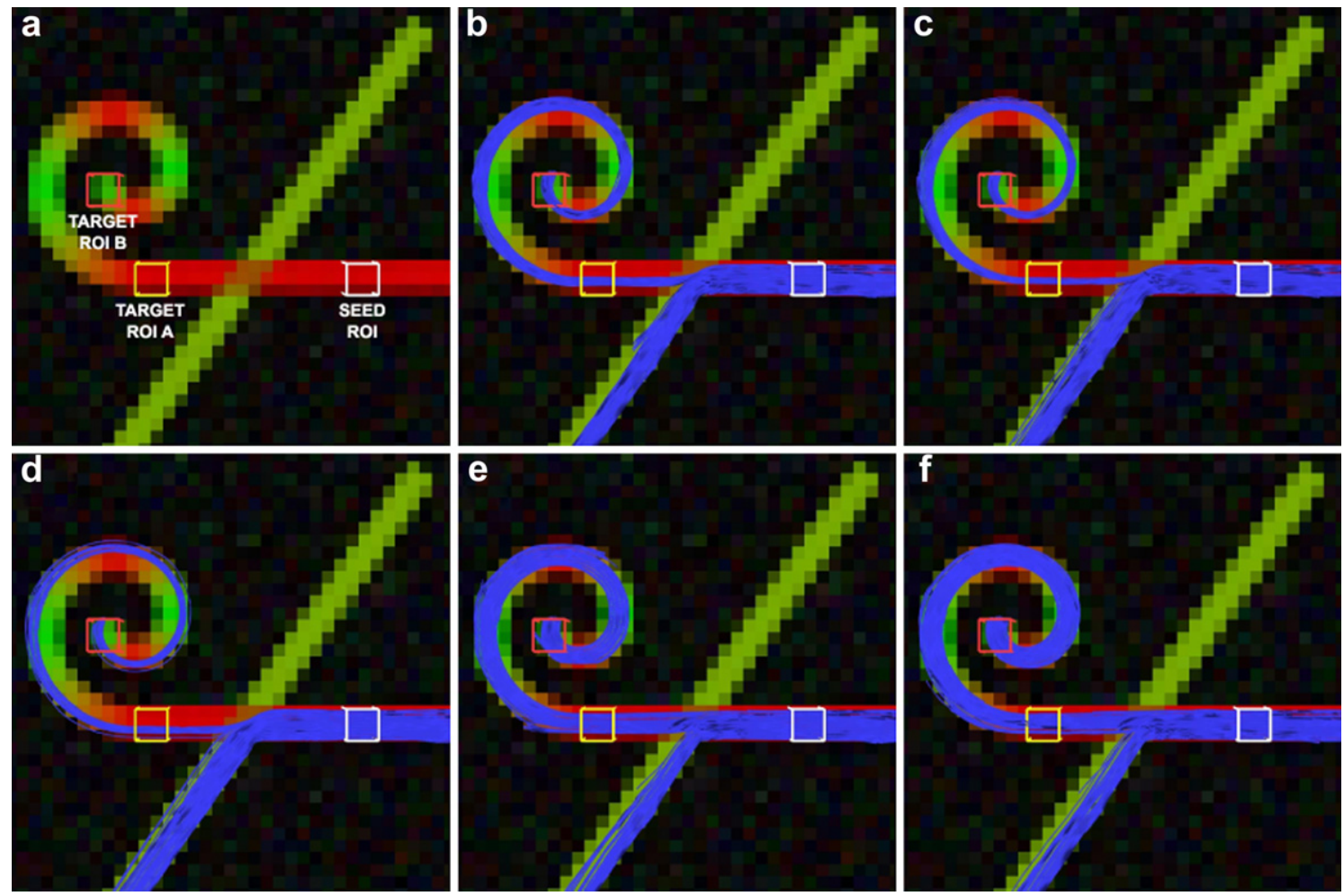

Figure 5 Results of multi-directional streamline tracking with a synthetic CFC dataset $\left(\alpha_{c}=55^{\circ}, d_{f}=1.0\right.$, SNR $\left.=35\right)$. In $(\mathrm{a})$, one cubic seed and two target ROls are marked by the white, yellow, and red boxes. In (b), tracking was performed with the unfiltered ODF dataset. Image (c) shows tracking results with local smoothing $\left(\sigma_{a}=9^{\circ}\right)$. In (d) ODFs were reconstructed with Laplace-Beltrami regularization $(\lambda=0.006)$. Fibers resulting from filtering the ODFs with ASRR $\left(\sigma_{a}=4.5^{\circ}\right)$ are shown in images $(e)$ and (f). Unlike in $(e)$, in image (f) ASRR was applied only to ODFs classified as multimodal (dynamic ASRR), resulting in a slightly better reconstruction of the spiral part. Overall, ASRR clearly increases the number of fibers reaching the target ROls. (For interpretation of the references to color in this figure legend, the reader is referred to the web version of this article.) 
at angles above $50^{\circ}$. Moreover, the accuracy of ASRR shows a much more stable behavior toward a crossing angle of $90^{\circ}$. (3) At $\alpha_{c}=90^{\circ}$, the accuracy of all methods is nearly equivalent. Only at $\mathrm{SNR}=10$ does ASRR show a slight advance. By contrast, the success rate is clearly improved by ASRR, especially when SNR is low. (4) The best angular smoothing value $\sigma_{a}$ for full ASRR seems to be $\sigma_{a}=\tilde{\sigma}_{a} / 2$. While $\sigma_{a}=\widetilde{\sigma}_{a} / 4$ results in a slightly higher success rate at low crossing angles in a range of $50-60^{\circ}$, $\sigma_{a}=\widetilde{\sigma}_{a} / 2$ seems to perform better at angles above $60^{\circ}$, which becomes obvious in particular at $\mathrm{SNR}=10$. On the other hand, the difference in accuracy between $\sigma_{a}=\widetilde{\sigma}_{a} / 4$ and $\sigma_{a}=\widetilde{\sigma}_{a} / 2$ is low.

\section{Evaluation of crossing fiber tractography}

On the basis of the results presented in the previous sections, we designed tractography experiments using synthetic CFC datasets. Within a cubic seed ROI placed before the region where both fibers cross, we generated 1000 seed points. For each seed point, a tract was generated using the streamline algorithm described in the Methods section. We then counted the number of tracts that passed through a target ROI A, located right behind the crossing region, and target ROI B, which was positioned in the inner termination of the spiral part of the fiber to be reconstructed. In Fig. 5, image (a) shows the ROI overlaid onto an FA slice that depicts the architecture of synthetic fibers crossing at an angle of $55^{\circ}$. The tracking experiment was performed for different combinations of $\alpha_{c}$, SNR, and $d_{f}$ (dataset configurations) and repeated under different conditions (ODF filters/ODF reconstructions):

1. Unfiltered $(\lambda=0)$

2. Local (intravoxel) smoothing $\left(\sigma_{r} \rightarrow 0 \mathrm{~mm}, \sigma_{a}=\widetilde{\sigma}_{a}, \lambda=0\right)$

3. Laplace-Beltrami regularization $(\lambda=0.006)$

4. ASRR $\left(\sigma_{r}=4 \mathrm{~mm}, \sigma_{a}=\widetilde{\sigma}_{a} / 2, \lambda=0\right)$

5. Dynamic ASRR (same parameters as ASRR)

Quantitative results are shown in Fig. 7. For small crossing angles $\left(\alpha_{c}=45^{\circ}, \alpha_{c}=55^{\circ}\right)$, ASRR clearly increases the number of fibers reaching both target ROI A and $B$. In Fig. 6, images (e) (ASRR) and (f) (dynamic ASRR) offer many more fibers that reach both target regions compared to the unfiltered ODF dataset (b), local smoothing (c), or Laplace-Beltrami regularization (d). When ASRR is not applied, fibers tend to deviate into the lower branch of the crossing, which is due to the fact that in the corresponding ODFs often only one maximum lying between the actual directions can be detected (images (b) $-(d)$ in Fig. 6).

At $\alpha_{c}=75^{\circ}$ and $d_{f}=1$, the success rate of ODF maxima detection is high for all methods. Here, the linearizing effect of ASRR begins to surpass its improvement in success rate and accuracy within the crossing region. While still slightly more tracts reach target ROI A when
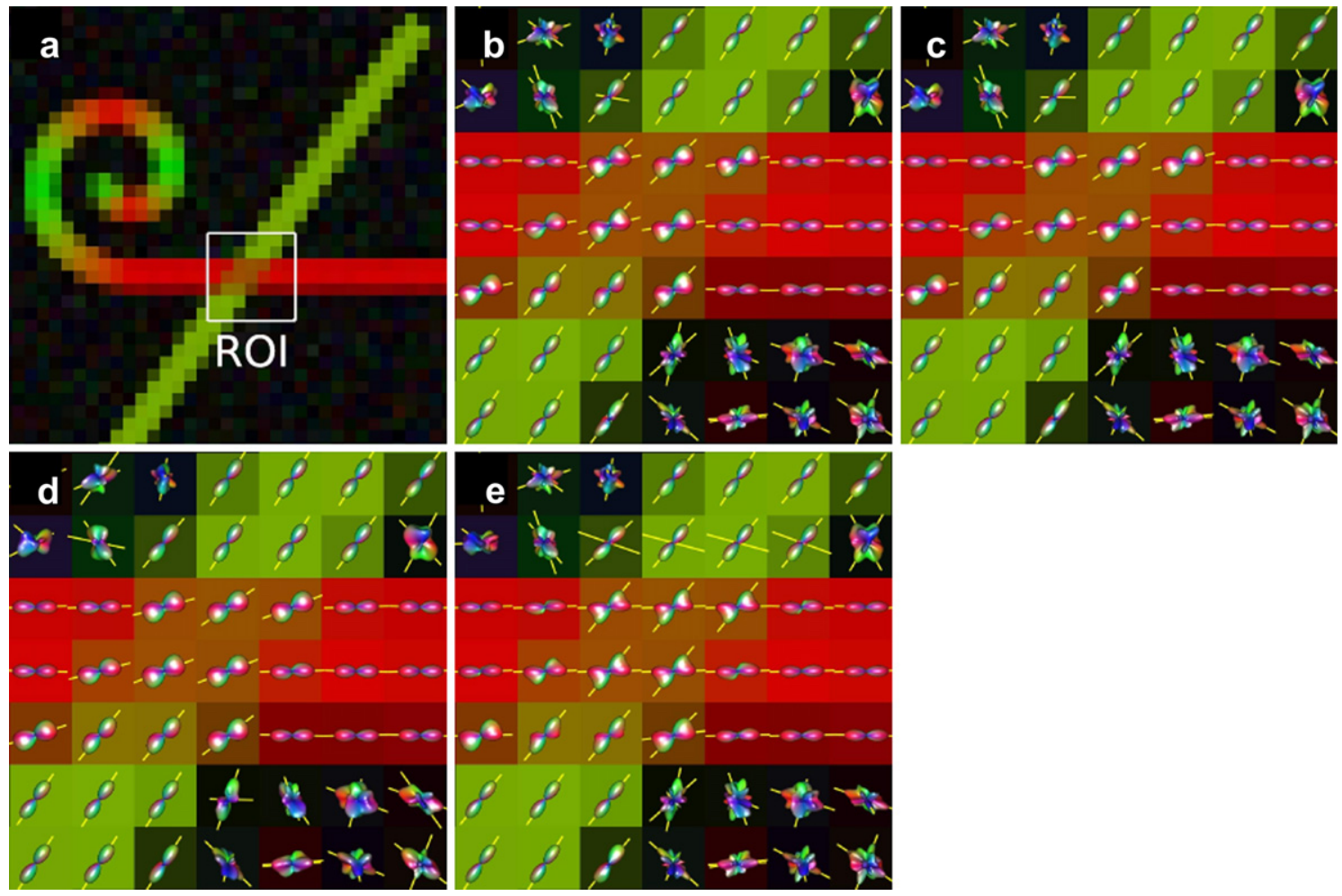

Figure 6 ODFs in the crossing region of a synthetic CFC dataset $\left(\alpha_{c}=55^{\circ}, d_{f}=1.0, \mathrm{SNR}=35\right)$. Detected ODF maxima are depicted by the yellow lines. Both fiber systems occupy equal volume fractions. In (a), the ROI used to select the ODFs for visualization is marked by the white box. In (b), unfiltered ODFs are shown. Image (c) shows ODFs with local smoothing $\left(\sigma_{a}=9^{\circ}\right)$. In (d) ODFs were reconstructed with Laplace-Beltrami regularization $(\lambda=0.006)$. As shown in image $(\mathrm{e})$, filtering the ODFs with ASRR $\left(\sigma_{a}=4.5^{\circ}\right)$ clearly improves the detection rate and accuracy of the fiber system orientations. (For interpretation of the references to color in this figure legend, the reader is referred to the web version of this article.) 

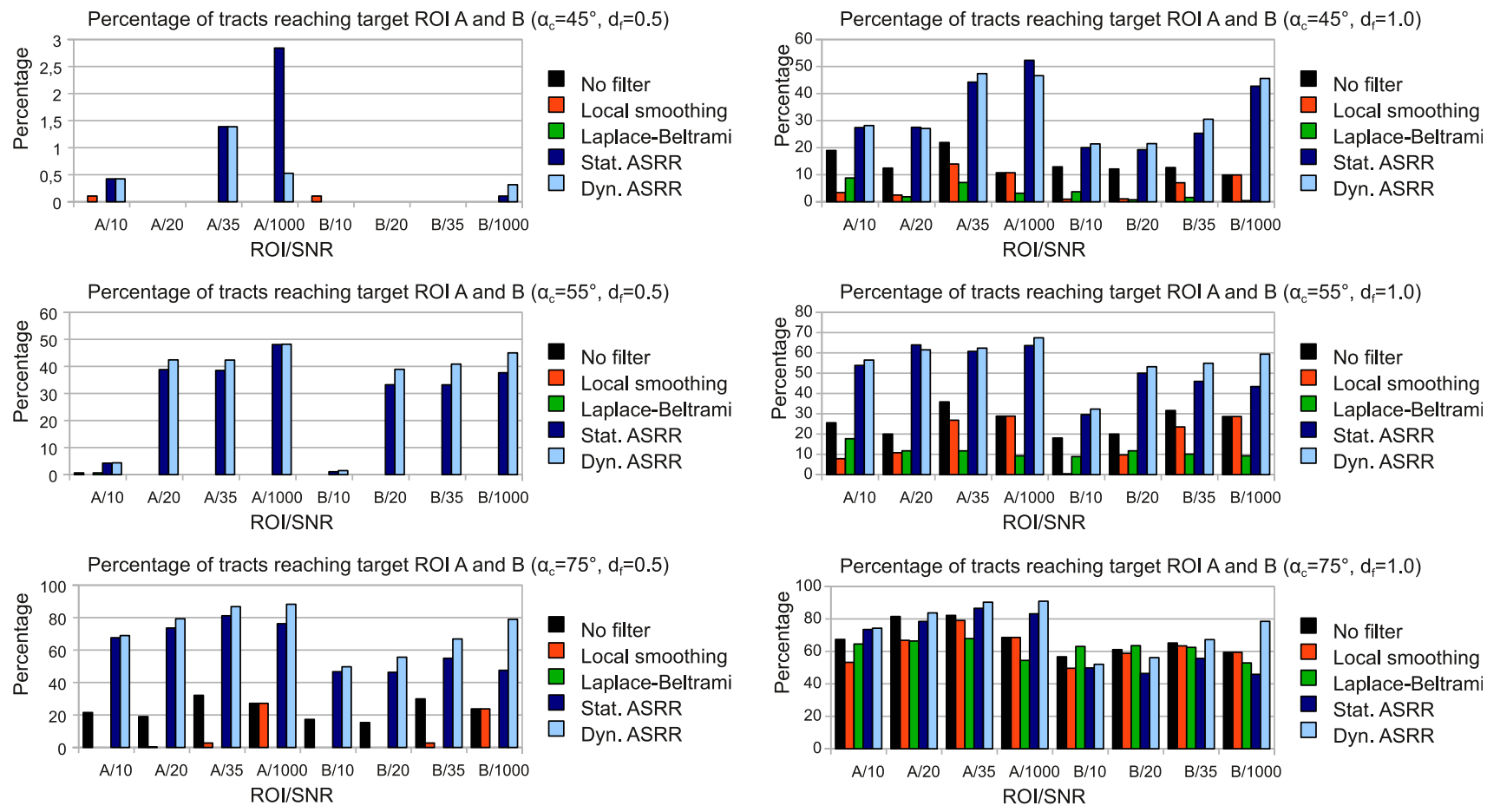

Figure 7 Percentage of tracts reaching target ROI A and B after multi-directional streamline tracking with synthetic CFC datasets of different configurations (SNR, fiber dominance ratio $d_{f}$, fiber-crossing angle $\alpha_{c}$ ).
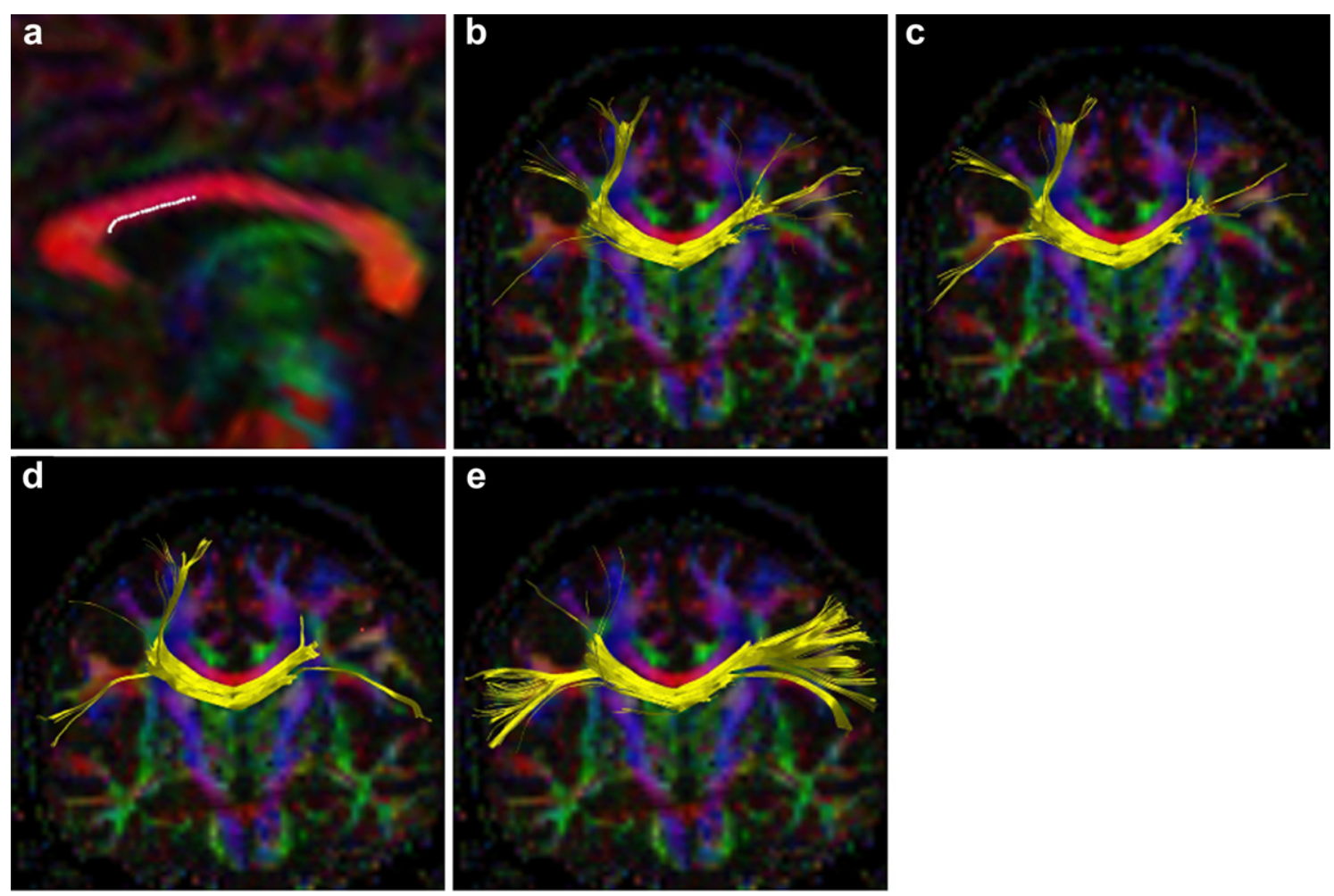

Figure 8 Multi-directional streamline tracking of the corpus callosum within a human brain. In order to reconstruct lateral transcallosal fibers, seed points were placed in the lower part of the genu on the mid-sagittal plane (a). In (b), ODFs were not filtered. Local smoothing $\left(\sigma_{a}=9^{\circ}\right)$ was applied in image (c). In image (d), ODFs were reconstructed with Laplace-Beltrami regularization $(\lambda=0.006)$. In $(\mathrm{e})$, dynamic ASRR $\left(\sigma_{a}=4.5^{\circ}\right)$ was applied to the ODFs prior to fiber tracking, which causes many more lateral transcallosal fibers to be revealed than by the other methods. 

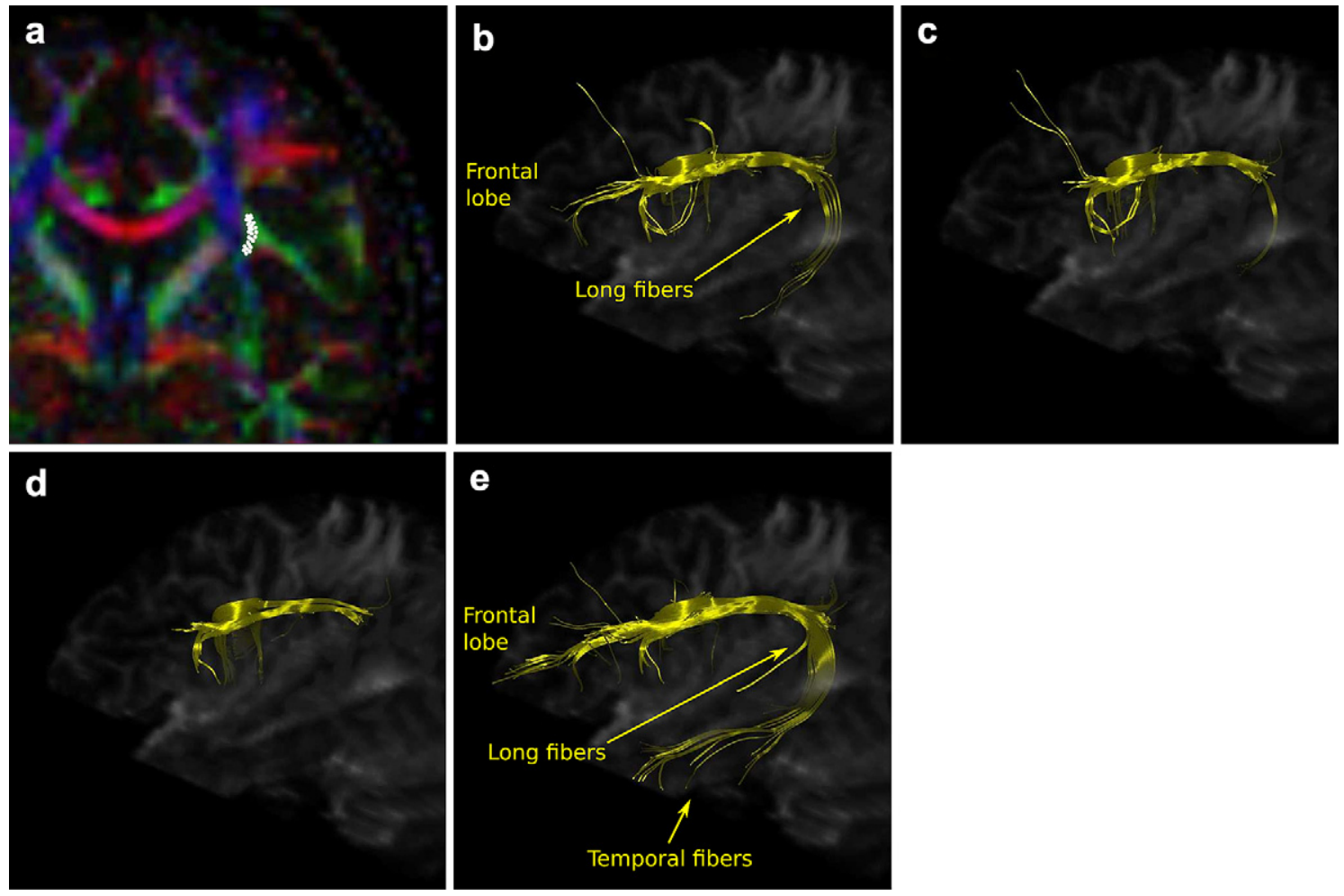

Figure 9 Multi-directional streamline tracking of the superior longitudinal fasciculus (SLF) within a human brain. Seed points were placed on a frontal plane inside the SLF (a). In (b), ODFs were not filtered. Local smoothing $\left(\sigma_{a}=9^{\circ}\right)$ was applied in image (c). In image (d), ODFs were reconstructed with Laplace-Beltrami regularization $(\lambda=0.006)$. In (e), dynamic ASRR $\left(\sigma_{a}=4.5^{\circ}\right)$ was applied to the ODFs prior to fiber tracking. Local smoothing and Laplace-Beltrami regularization seem to prevent the long fibers of the SLF from being reconstructed in full. Compared to the unfiltered dataset, dynamic ASRR reveals many more of the long fibers.

ASRR is applied, the reconstruction of the tightly curved spiral part has deteriorated, which can be seen in the decreased number of tracts that reach target ROI $B$. The results also show that local smoothing does not seem to improve tracking at fiber-crossing angles below $75^{\circ}$ in such an experimental setting. Again, as observed in the experiments discussed in the previous section, low SNR eases the satisfaction of the local maximum constraint, which causes tracking with local smoothing to slightly fall behind and demonstrates the weakness of the method used to detect diffusion peaks. Apart from these findings, ASRR considerably improves tracking results at all three crossing angles in case the fiber to be reconstructed is dominated by the crossing fiber $\left(d_{f}=0.5\right)$. If we apply ASRR to ODFs classified as multimodal only, the reconstruction of the spiral part can be enhanced. Since the classification performance decreases with SNR, the advance of dynamic ASRR over static ASRR maximizes at $\mathrm{SNR}=1000$.

\section{Human subject study}

We employed multi-directional streamline tracking in order to evaluate ASRR in the reconstruction of two distinct fiber systems in the human brain: (1) the corpus callosum (CC) and (2) the superior longitudinal fasciculus (SLF).

In the corpus callosum, we tried to delineate commissural fibers connecting lateral regions of both hemispheres.
While the reconstruction of the known c-shaped part is not a problem even with DTI, lateral fibers run through regions where they cross densely packed fibers from, e.g., portions of the corticospinal tract. Thus, we manually placed 25 seed points inside the lower part of the genu on the midsagittal plane (see Fig. 8, image a). For each seed point, 80 additional seed points were generated randomly inside a radius of $0.5 \mathrm{~mm}$, leading to a total of 2025 seed points. As it can be seen in Fig. 8, image (e), the reconstruction of lateral transcallosal fibers succeeds best with dynamic ASRR. Local smoothing as depicted in image (c) does not improve tracking results compared to the unfiltered ODF dataset, which was used in image (b). Laplace-Beltrami regularization as shown in image (d) seems to be of only slight advantage.

The superior longitudinal fasciculus is a long bundle of neurons connecting the frontal cortex with the occipital and parietal association areas of the cerebrum. Being part of the long fibers, some neurons radiate into the occipital lobe, while others turn in a great arc around the putamen to the anterior regions of the temporal lobe. We placed 27 seed points (Fig. 9, image a) on a coronal plane inside the cross section of the SLF. For each seed point, 10 additional seed points were generated randomly inside a radius of $1 \mathrm{~mm}$. Thus, we performed tracking with a total of 297 seed points. Fig. 9 shows that local smoothing (image c) and Laplace-Beltrami regularization (image d) seem to prevent the long fibers from being reconstructed completely. Tracking with the unfiltered dataset generates more of 

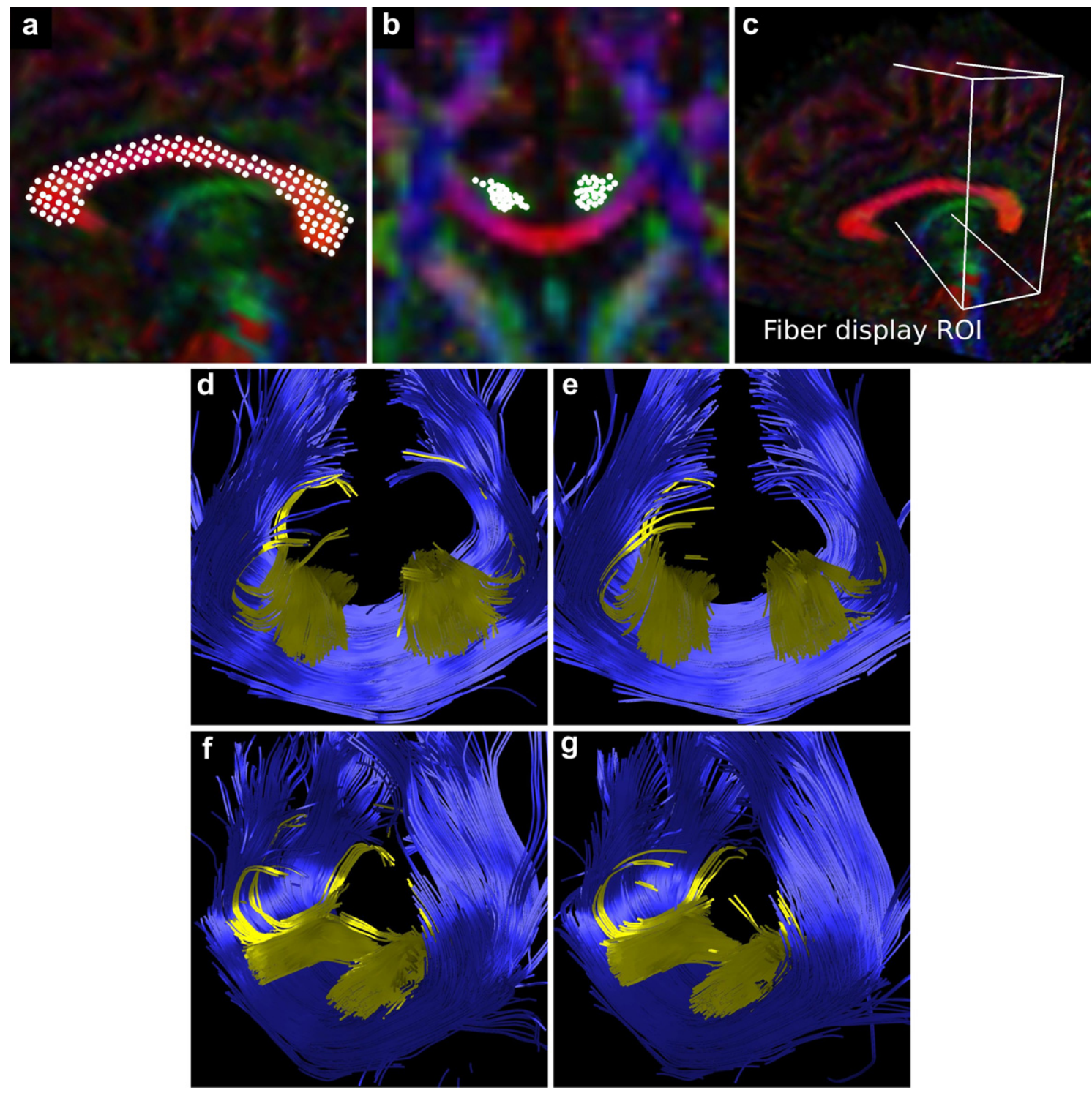

Figure 10 Multi-directional streamline tracking of the corpus callosum and the cingulum within the human subject dataset. In order to study partial voluming at the boundary between both fiber systems, seed points were placed inside a mid-sagittal slice through the CC (a) and a coronal slice through the cingulum (b). For visual inspection, fibers were masked by a rectangular region (c). It is obvious that a small portion of the cingular (yellow) fibers deviate into the callosal (blue) fibers. However, ASRR (e, g) does not seem to amplify this effect compared to the results obtained without filtering $(d, f)$.
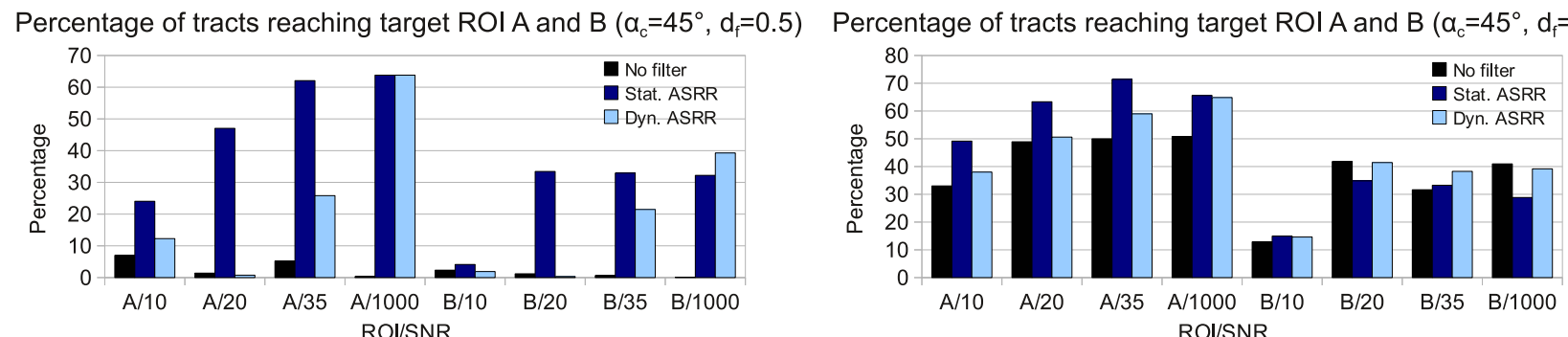

Figure 11 Percentage of tracts reaching target ROI A and B after multi-directional streamline tracking with a synthetic CFC dataset $\left(\alpha_{c}=45^{\circ}\right)$ of different configurations (SNR, fiber dominance ratio $d_{f}$ ). ODFs were reconstructed with Constant Solid Angle (CSA) [12] with an $\mathrm{SH}$ order of 8. 
them, but dynamic ASRR brings out a much larger portion of both the superior and the inferior part of these fibers. Also, more of the temporal fibers and those originating in the frontal lobe can be viewed.

Parameters we chose were $\sigma_{a}=9^{\circ}$ for local smoothing and $\sigma_{a}=4.5^{\circ}$ for ASRR.

\section{Conclusion and discussion}

We have presented a straightforward yet effective method for the smoothing, regularization, and sharpening of diffusion profiles in crossing areas of ODF fields obtained from diffusion data acquired under the imaging restrictions of the clinical routine. In order to increase the accuracy of ODF maxima detection and to avoid smoothing out nondominant diffusion peaks, ASRR takes into account information from neighboring voxels, thus combining both a classical intravoxel and an advanced intervoxel processing strategy. Since the method encompasses both an angular and a radial smoothing kernel, it possesses a high degree of generality, and its two parameters $\sigma_{a}$ and $\sigma_{r}$ may be adapted to specific datasets and applications.

On the basis of the quantitative analyses with synthetic datasets, we can conclude that it was possible to improve the accuracy of detected ODF maxima through ASRR. The potential of ASRR to strengthen signals of non-dominant fiber systems could also be clearly demonstrated. Tracking experiments with data from a healthy human subject, acquired under clinical imaging conditions, showed the method's potential to notably optimize the reconstruction of lateral transcallosal fibers and the long fibers of the superior longitudinal fasciculus.

The tendency of ASRR to linearize trajectories in tightly curved fiber systems such as the spiral part of the synthetic CFC dataset could be compensated largely with dynamic ASRR. However, in situations with low SNR $(10,20)$ and higher fiber-crossing angles $\left(\geq 75^{\circ}\right)$, it could be observed that the linearizing effect begins to surpass the benefits of regularization and sharpening if the fiber to be reconstructed is not dominated by a crossing fiber. Otherwise, ASRR regains its advantage.

The further reduction of the linearizing effect will be a subject of future work. For example, more reliable techniques of ODF classification with better robustness to noise may be elaborated to advance dynamic ASRR. Also, the point symmetry of ODFs filtered with ASRR could be abandoned, leading to profiles which reflect spatial neighborhood by different diffusion probabilities along and against a reconstruction direction. While this is conceivable to compensate linearizing, it constitutes a concept that is rather uncommon in diffusion tractography and at least demands a separate and detailed investigation, which is beyond the scope of this paper. Nevertheless, we like to mention that such a concept has been investigated by [26], but in the context of splaying rather than crossing fibers. Of course, choosing alternative spatial filter kernels may also improve the results.

Regarding the parameterization of ASRR, it has been shown that using a half or a quarter of $\widetilde{\sigma}_{a}$ for angular smoothing promises the best results. When we choose $\sigma_{a} \rightarrow 0^{\circ}$, angular smoothing is not performed explicitly, which may be appropriate if ODFs are smooth enough. Note that tri-linear interpolation already induces a smoothing effect to the ODFs. Therefore, for noise suppression, we do not regard angular smoothing to be mandatory in any case. At the other hand, choosing $\sigma_{a} \rightarrow 0^{\circ}$ causes the shape of the filter kernel to degenerate to a thin beam, which increases the linearizing effect. Choosing a value of $\sigma_{r}$ that is too high will also amplify linearizing. The parameter $\sigma_{r}$ should be chosen proportionally to the diameter of the crossing regions expected, which was $5 \mathrm{~mm}$ in our synthetic datasets. We chose a slightly smaller value of $\sigma_{r}=4 \mathrm{~mm}$ because we found this value to be a good tradeoff between increased accuracy within crossing regions and decreased accuracy within curves falling victim to the linearizing tendency. The extent of the crossing region to which ASRR can successfully be applied is certainly restricted. The determination of the exact limit requires many more simulation studies under different conditions and parameterizations. Also, the work presented here does not investigate fiber architectures where tightly bending fibers cross. On the other hand, synthetic data can reproduce only a subset of features that influence measurements taken under real conditions, e.g., motion shift, distortions at boundaries, ghosting, or vibration. Thus, the examination of additional fiber systems on a broader variety of in vivo datasets would support the evaluation of ASRR.

We would also like to point out that methods that incorporate information from neighborhood may increase the influence of partial volume effects. Since our method does not explicitly handle boundaries between fiber systems that run near by, the creation of non-existent crossings or deviations into neighboring fiber systems cannot generally be excluded. Fig. 10 shows fibers from the corpus callosum and the cingulum obtained with deterministic tracking similar to the experiments presented above. Both fiber systems run very close to each other and deviations from the cingulum into the callosal fibers due to partial voluming can be observed. However, in this case ASRR (images $(e, g)$ ) does not seem to enhance this effect compared to the results obtained without filtering (images $(d, f)$ ).

Regarding model-based methods such as tensor decomposition and spherical deconvolution or ODF reconstruction with CSA, which promise a more accurate detection of fiber orientations inside multimodal voxels but also decrease in performance in the presence of noise or partial voluming, it would be interesting to see whether ASRR could assist. Fig. 11 shows preliminary results from a tracking experiment with the CFC dataset on the basis of CSA-ODFs of order 8. At a fiber crossing angle of $\alpha_{c}=45^{\circ}$, tracking through the crossing region (target region $A$ ) is still improved by ASRR. However, the linearizing effect of ASRR is increased in the presence of the sharp CSA-ODFs and the effect of dynamic ASRR is diminished, which is due to the classification method not being adapted to the noisy CSAODFs. For those approaches working with raw diffusion data, the impact of ASRR could be transferred to the diffusion signals with the help of the inverse FRT applied to the filtered ODFs. Since in the framework of spherical harmonics the FRT reduces to a matrix multiplication [11], the inverse matrix can be used to recompute artificial gradient signals. It would also be interesting to compare our 
method, being applied after ODF reconstruction, to the recent work of Reisert et al. [19], which uses a neighborhood-based regularizer prior to the computation of a fiber ODF obtained with constrained spherical deconvolution. In light of the effort required by the investigations mentioned here, we have to leave them to future work.

Finally, we would like to mention that the concept of taking into account samples from a regional neighborhood may also be used in the design of tractography algorithms in order to better estimate the most probable propagation direction. It has been demonstrated that this can help to solve ambiguities still existent in ODFs in which noise has been reduced by intravoxel smoothing $[27,28]$. Nevertheless, since ASRR works at a more general stage of the processing chain, we hope that our method may contribute to improving crossing fiber tractography in general.

\section{References}

[1] Tuch DS, Reese TG, Wiegell MR, Makris N, Belliveau JW, Wedeen VJ. High angular resolution diffusion imaging reveals intravoxel white matter fiber heterogeneity. Magn Reson Med 2002;48(4):577-82.

[2] Basser PJ, Mattiello J, LeBihan D. MR diffusion tensor spectroscopy and imaging. Biophys J 1994;66(1):259-67.

[3] Behrens TEJ, Woolrich MW, Jenkinson M, Johansen-Berg $H$, Nunes RG, Clare $S$, et al. Characterization and propagation of uncertainty in diffusion-weighted MR imaging. Magn Reson Med 2003;50(5):1077-88.

[4] Schultz T, Seidel HP. Estimating crossing fibers: a tensor decomposition approach. IEEE Trans Vis Comput Graph 2008; 14(6):1635-42.

[5] Tuch DS. Q-ball imaging. Magn Reson Med 2004;52(6):1358-72.

[6] Jansons KM, Alexander DC. Persistent angular structure: new insights from diffusion MRI data. Inf Process Med Imaging 2003; 18:672-83.

[7] Anderson AW. Measurement of fiber orientation distributions using high angular resolution diffusion imaging. Magn Reson Med 2005;54(5):1194-206.

[8] Tournier JD, Calamante F, Connelly A. Robust determination of the fibre orientation distribution in diffusion MRI: nonnegativity constrained super-resolved spherical deconvolution. Neuroimage 2007;35(4):1459-72.

[9] Descoteaux M, Deriche R, Knösche TR, Anwander A. Deterministic and probabilistic tractography based on complex fibre orientation distributions. IEEE Trans Med Imaging 2009;28(2): 269-86.

[10] Hess CP, Mukherjee P, Han ET, Xu D, Vigneron DB. Q-ball reconstruction of multimodal fiber orientations using the spherical harmonic basis. Magn Reson Med 2006;56(1):104-17.

[11] Descoteaux M, Angelino E, Fitzgibbons S, Deriche R. Regularized, fast, and robust analytical q-ball imaging. Magn Reson Med 2007;58(3):497-510.

[12] Aganj I, Lenglet C, Sapiro G, Yacoub E, Ugurbil K, Harel N. Reconstruction of the orientation distribution function in single- and multiple-shell q-ball imaging within constant solid angle. Magn Reson Med 2010;64(2):554-66.
[13] Tristán-Vega A, Westin CF, Aja-Fernández S. Estimation of fiber orientation probability density functions in high angular resolution diffusion imaging. Neuroimage 2009;47(2):638-50.

[14] Canales-Rodríguez EJ, Melie-García L, Iturria-Medina Y. Mathematical description of q-space in spherical coordinates: exact q-ball imaging. Magn Reson Med 2009;61(6):1350-67.

[15] Zhan W, Yang Y. How accurately can the diffusion profiles indicate multiple fiber orientations? A study on general fiber crossings in diffusion MRI. J Magn Reson 2006;183(2):193-202.

[16] Savadjiev P, Campbell JSW, Pike GB, Siddiqi K. 3D curve inference for diffusion MRI regularization. Med Image Comput Comput Assist Interv Int Conf Med Image Comput Comput Assist Interv 2005;8(Pt 1):123-30.

[17] Savadjiev P, Campbell JSW, Pike GB, Siddiqi K. 3d curve inference for diffusion MRI regularization and fibre tractography. Med Image Anal 2006;10(5):799-813.

[18] Goh A, Lenglet C, Thompson PM, Vidal R. Estimating orientation distribution functions with probability density constraints and spatial regularity. Med Image Comput Comput Assist Interv 2009;12(Pt 1):877-85.

[19] Reisert M, Kiselev VG. Fiber continuity: an anisotropic prior for ODF estimation. IEEE Trans Med Imaging 2011;30(6): 1274-83.

[20] Duits R, Franken E. Left-invariant diffusions on the space of positions and orientations and their application to crossingpreserving smoothing of HARDI images. Int J Comp Vis 2010; 90.

[21] Otto KM, Ehricke HH, Kumar V, Klose U. Regularization of diffusion MRI q-ball fields for crossing fiber tractography. In: World congress on medical physics and biomedical engineering, September 7-12, 2009, Munich, Germany. Berlin, Heidelberg: Springer; 2009. p. 845-8. vol. 25 of IFMBE Proceedings.

[22] Ehricke HH, Otto KM, Kumar V, Klose U. Diffusion MRI tractography of crossing fibers by cone-beam ODF regularization. In: Proceedings of the 31st DAGM symposium on pattern recognition. Berlin, Heidelberg: Springer-Verlag; 2009. p. 412-21.

[23] Behrens TEJ, Berg HJ, Jbabdi S, Rushworth MFS, Woolrich MW. Probabilistic diffusion tractography with multiple fibre orientations: what can we gain? Neuroimage 2007;34(1):144-55.

[24] Wedeen VJ, Wang RP, Schmahmann JD, Benner T, Tseng WYI, Dai $G$, et al. Diffusion spectrum magnetic resonance imaging (DSI) tractography of crossing fibers. Neuroimage 2008;41(4): 1267-77.

[25] Alexander DC, Barker GJ, Arridge SR. Detection and modeling of non-gaussian apparent diffusion coefficient profiles in human brain data. Magn Reson Med 2002;48(2):331-40.

[26] Barmpoutis A, Vemuri BC, Howland D, Forder JR. Extracting tractosemas from a displacement probability field for tractography in DW-MRI. Med Image Comput Comput Assist Interv 2008;11(Pt 1):9-16.

[27] Savadjiev P, Campbell JSW, Descoteaux M, Deriche R, Pike GB, Siddiqi K. Labeling of ambiguous subvoxel fibre bundle configurations in high angular resolution diffusion MRI. Neuroimage 2008;41(1):58-68.

[28] Tournier JD, Calamante F, Connelly A. Improved probabilistic streamlines tractography by second order integration over fibre orientation distributions. Proc Intl Soc Mag Reson Med 2010;18:1670. 